\title{
Original ARticle \\ Obstructive sleep apnea and longitudinal Alzheimer's disease biomarker changes
}

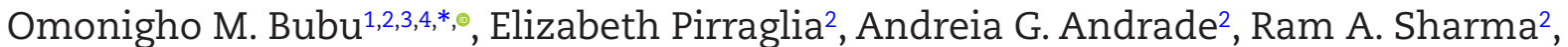 \\ Sandra Gimenez-Badia, ${ }^{5, \bullet}$, Ogie Q. Umasabor-Bubu ${ }^{6}$, Megan M. Hogan³, Amanda M. Shim³, \\ Fahad Mukhtar ${ }^{1}$, Nidhi Sharma ${ }^{7}$, Alfred K. Mbah ${ }^{1}$, Azizi A. Seixas' ${ }^{4}$, Korey Kam ${ }^{8, \bullet}$, \\ Ferdinand Zizi ${ }^{4}$, Amy R. Borenstein ${ }^{9}$, James A. Mortimer ${ }^{1}$, Kevin E. Kip ${ }^{1}$, David Morgan ${ }^{10,11}$, \\ Ivana Rosenzweig ${ }^{12,13}$, Indu Ayappa ${ }^{8}$, David M. Rapoport ${ }^{8}$, Girardin Jean-Louis', Andrew W. \\ $\operatorname{Varga}^{8,0}$, and Ricardo S. Osorio ${ }^{2,14, *}$ for the Alzheimer's Disease Neuroimaging Initiative
}

${ }^{1}$ Department of Epidemiology and Biostatistics, College of Public Health, University of South Florida, Tampa, FL, ${ }^{2}$ Center for Brain Health, Department of Psychiatry, NYU Langone Medical Center, New York, NY, ${ }^{3}$ Department of Applied Health Sciences, Wheaton College, Wheaton, IL, ${ }^{4}$ Department of Population Health, New York University School of Medicine, Center for Healthful Behavior Change, New York, NY, ${ }^{5}$ Multidisciplinary Sleep Unit - Respiratory Department, Hospital de la Santa Creu i Sant Pau. IIB Sant Pau Department of Clinical Psychobiology, University of Barcelona, Barcelona, Spain, ${ }^{6}$ State University New York (SUNY) Downstate Medical Center, Brooklyn, NY, ${ }^{7}$ Dipartmento Di Scienze Biochimica A. Rossi Fanelli, University of Rome, Rome, Italy, ${ }^{8}$ Division of Pulmonary, Critical Care and Sleep Medicine at the Icahn School of Medicine at Mount Sinai, New York, NY, ${ }^{9}$ Department of Family Medicine and Public Health, University of California-San Diego, San Diego, CA, ${ }^{10}$ Department of Molecular Science and Translational Medicine, Michigan State University, Grand Rapids, MI, ${ }^{11}$ Byrd Alzheimer's Disease Institute, College of Medicine, Psychiatry and Behavioral Neurosciences, University of South Florida Health, Tampa, FL, ${ }^{12}$ Sleep Disorders Centre, Guy's Hospital, Great Maze Pond, London, UK, ${ }^{13}$ Sleep and Brain Plasticity Centre, Department of Neuroimaging, IoPPN, King's College London, London, UK and ${ }^{14}$ Nathan S. Kline Institute for Psychiatric Research, Orangeburg, NY

*Corresponding authors. Omonigho M. Bubu, 180 Madison Avenue, 7th Floor, New York, NY 10016. Email: omonigho.bubu@nyulangone.org.; Ricardo S. Osorio, 145 East 32nd Street, 5th Floor, New York, NY 10016-6055. Email: ricardo.osorio@nyumc.org.

\begin{abstract}
Study Objectives: To determine the effect of self-reported clinical diagnosis of obstructive sleep apnea (OSA) on longitudinal changes in brain amyloid PET and CSF biomarkers (AB42, T-tau, and P-tau) in cognitively normal (NL), mild cognitive impairment (MCI), and Alzheimer's disease (AD) elderly.

Methods: Longitudinal study with mean follow-up time of $2.52 \pm 0.51$ years. Data were obtained from the Alzheimer's Disease Neuroimaging Initiative (ADNI) database. Participants included $516 \mathrm{NL}, 798 \mathrm{MCI}$, and $325 \mathrm{AD}$ elderly. Main outcomes were annual rate of change in brain amyloid burden (i.e. longitudinal increases in florbetapir PET uptake or decreases in CSF A 42 levels); and tau protein aggregation (i.e. longitudinal increases in CSF total tau [T-tau] and phosphorylated tau [P-tau]). Adjusted multilevel mixed effects linear regression models with randomly varying intercepts and slopes was used to test whether the rate of biomarker change differed between participants with and without OSA.

Results: In NL and MCI groups, OSA+ subjects experienced faster annual increase in florbetapir uptake $(\mathrm{B}=.06,95 \% \mathrm{CI}=.02, .11$ and $\mathrm{B}=.08,95 \% \mathrm{CI}=.05, .12$, respectively) and decrease in CSF A 42 levels $(B=-2.71,95 \% \mathrm{CI}=-3.11,-2.35$ and $B=-2.62,95 \% \mathrm{CI}=-3.23,-2.03$, respectively); as well as increases in CSF T-tau $(B=3.68,95 \% \mathrm{CI}=3.31,4.07$ and $B=2.21,95 \% \mathrm{CI}=1.58,2.86$, respectively) and $\mathrm{P}$-tau $(B=1.221,95 \% \mathrm{CI}=1.02,1.42$ and $\mathrm{B}=1.74,95 \% \mathrm{CI}=1.22,2.27$, respectively); compared with OSA- participants. No significant variations in the biomarker changes over time were seen in the AD group.

Conclusions: In both NL and MCI, elderly, clinical interventions aimed to treat OSA are needed to test if OSA treatment may affect the progression of cognitive impairment due to $\mathrm{AD}$.
\end{abstract}

\section{Statement of Significance}

Recent studies show that obstructive sleep apnea (OSA) is associated with increased Alzheimer's disease (AD) risk. This study adds to the literature by providing evidence that OSA is related to longitudinal increases in amyloid and tau burden in cognitively normal and mild cognitive impairment (MCI) OSA patients when compared with healthy controls. These novel findings are directly relevant to the emerging literature examining for evidence of a causal relationship between OSA and $\mathrm{AD}$, and are of interest to further understand the various possible mechanistic links that explain this relationship. More importantly, it suggests that clinical interventions aimed to treat OSA in the elderly may slow the progression of cognitive impairment due to AD.

Key words: obstructive sleep apnea; Alzheimer's disease; longitudinal study; CSF biomarkers; brain amyloid PET; Aß42; T-tau; P-tau

Submitted: 12 October, 2018; Revised: 11 January, 2019

(c) Sleep Research Society 2019. Published by Oxford University Press on behalf of the Sleep Research Society.

All rights reserved. For permissions, please e-mail journals.permissions@oup.com. 


\section{Introduction}

Intermittent hypoxia and sleep fragmentation have been implicated as possible mechanistic links in a causal pathway between obstructive sleep apnea (OSA) and Alzheimer's disease (AD) [1-4]. Recent cross-sectional studies have demonstrated associations between $\mathrm{OSA}$ and $\mathrm{AD}$ biomarkers (i.e. brain amyloid PET and CSF biomarkers [A $\beta 42, T$-tau, and P-tau]) in both cognitively normal (NL) and mild cognitive impairment (MCI) elderly [5-7]. Clearly establishing whether OSA individuals are at heightened risk to develop $\mathrm{AD}$ is critical for preventing $\mathrm{AD}$. Positron emission tomography (PET) amyloid tracer uptake and decreases in cerebrospinal fluid (CSF) amyloid-beta42 (A $\beta 42)$ levels are robust predictors of amyloid burden as well as of future development of $\mathrm{AD}[8,9]$. Significant increases in CSF P-tau and CSF T-tau are also documented in AD patients compared with controls [10]. An important question is whether OSA-AD association is related to changes in AD neuropathology over time. Recently, our group demonstrated that objectively measured OSA was associated with markers of increased amyloid burden over a 2-year follow-up in the NYU cohort which consists exclusively of community-dwelling healthy cognitively normal elderly. In this study, we examined self-reported clinical diagnosis of OSA's association with longitudinal changes of brain amyloid burden and tau protein aggregation in a sample of cognitively normal (NL), mild cognitive impairment (MCI) and Alzheimer's disease (AD) elderly in the larger Alzheimer's Disease Neuroimaging Initiative (ADNI) cohort, to provide additional evidence for a possible causal relationship between OSA and AD.

\section{Methods}

Data were obtained from the ADNI database (adni.loni.usc.edu). ADNI was launched in 2003 as a public-private partnership, led by Michael W. Weiner, MD. The primary goal of ADNI has been to test whether serial MRI, PET, other biological markers, as well as clinical and neuropsychological assessments can be combined to measure the progression of $\mathrm{MCI}$ and early AD. Thus far, ADNI has recruited over 2,000 adults aged 55-90, consisting of NL, $\mathrm{MCI}$, and early AD. Follow-up for CSF sampling and PET typically occurs every 1-2 years.

\section{Standard protocol approvals, registrations, and patient consent}

The Institutional Review Board at each of the ADNI participating centers approved the ADNI study. Written informed consent was obtained from all patients participating in ADNI. ADNI inclusion and exclusion criteria are detailed elsewhere (http:// adni.loni.ucla.edu/wp-content/uploads/ 2010/09/ADNI_ GeneralProceduresManual.pdf).

\section{Study participants}

Participant data used for this study were based on medical history obtained from the ADNI database on December 2016. This study included 1,639 participants: $516 \mathrm{NL}, 798 \mathrm{MCI}$, and 325 AD. Subjects missing biomarker data were excluded. Subjects with preexisting comorbid sleep disorders other than OSA, and those with body mass index (BMI) change greater than 5 between visits, were further excluded, since both could modify group allocation (i.e. OSA+ vs. OSA-) [11]. Other exclusions included previous OSA surgery and having a reversible diagnosis (i.e. had a $\mathrm{MCI}$ or $\mathrm{AD}$ diagnosis at any time point but a NL diagnosis or MCI diagnosis, respectively, at their last visit), thereby allowing us to exclude reversible conditions and unspecified diagnoses.

\section{OSA diagnosis}

Presence or absence of OSA was based on self-reported clinical diagnosis of OSA (variable name: MHDESC) during a clinical interview. Patients with reported "sleep apnea," "sleep disordered breathing," "OSA," or "SDB" were labeled OSA+ and the remaining participants were considered OSA-. To ensure that patients were allocated into the correct groups, three physicians (R.O., S.A., and O.B.) reviewed medical history clinical notes from the ADNI download, for group allocation.

\section{NL, MCI, and AD diagnosis}

ADNI criteria for subject classification are described elsewhere [12]. NL and MCI subjects scored between 24 and 30 on the MiniMental State Examination (MMSE) while AD subjects scored between 20 and 26. MCI and AD participants had global Clinical Dementia Rating (CDR) scores of 0.5 and 1, respectively. The diagnosis of AD was made using established clinical criteria [13].

\section{Florbetapir PET imaging acquisition and interpretation}

ADNI florbetapir summary data was uploaded to the Laboratory of Neuroimaging (LONI) by the University of California at Berkeley [14, 15]. For more information on the methods, see https://adni.loni.usc.edu/wp-content/uploads/2010/05/ ADNI2_PET_Tech_Manual_0142011.pdf, http://adni.loni.usc. edu/updated-florbetapir-av-45-pet-analysis-results/. Briefly, native-space segmented and parcellated magnetic resonance imaging (MRI) scan with Freesurfer (version 5.3.0) was used for each subject to define cortical gray matter regions of interest (frontal, anterior/posterior cingulate, lateral parietal, lateral temporal) that make up a summary cortical region of interests (ROI). Five reference regions (cerebellar gray matter, whole cerebellum, brainstem/pons, eroded subcortical white matter, and a composite reference region) were defined. Each florbetapir scan was then matched to the corresponding MRI and the mean florbetapir uptake within the cortical and reference regions is calculated. The baseline MRI was used to define regions for all subsequent florbetapir scans. Image data are available at three levels of preprocessing (raw, unsmoothed, and smoothed) as described online at http://adni.loni.usc.edu/data-samples/ $\mathrm{mri} /$. Calculation of florbetapir standardized uptake value ratios (SUVRs) were done by obtaining means across the four cortical regions and dividing this cortical summary ROI by one of the five reference regions. Cortical ROI selection and reference region is predicated on the goals of the analysis. Two summary SUVRs were provided. The first is the cortical summary ROI divided by the whole cerebellum reference region (SUMMARYSUVR_WHOLECEREBNORM), which as recommended we used for cross-sectional florbetapir analyses. The second is 
the cortical summary ROI divided by a composite reference region (SUMMARYSUVR_COMPOSITE_REFNORM), which as recommended we used for our longitudinal florbetapir analyses.

\section{Cerebrospinal fluid methods}

CSF biospecimen data collection details can be found at http:// adni.loni.usc.edu/data-samples/biospecimen-data/. Briefly, A 442 , T-tau, and P-tau181 were measured from CSF that was collected at each ADNI site, and transferred into polypropylene transfer tubes followed by freezing on dry ice within $1 \mathrm{~h}$ after collection and shipped overnight to the ADNI Biomarker Core laboratory at the University of Pennsylvania Medical Centre on dry ice. A standardized protocol was implemented to quantify biomarker concentrations in each of the CSF baseline aliquots using a multiplex xMAP Luminex platform (Luminex Corp, Austin, TX) with Innogenetics (INNO-BIA AlzBio3, Ghent, Belgium; for research use only reagents) immunoassay kitbased reagents, validated in Vanderstichele et al. [16] and Shaw et al. [17]. Further details can be found at http://www.adni-info. org/index.php.

\section{Covariates/potential confounders}

As our study tried to answer an etiological question, we employed a broad selection of covariates based on published literature $[18,19]$. Covariates were selected a priori and included factors that might be more common in cases with OSA and that might contribute to either A $\beta$-pathology (CSF A $\beta 42$ and florbetapir $\mathrm{PET}$ ) or neurodegeneration (CSF T-tau), including age, sex, BMI, education, CPAP use, ApoE4 status, alcohol intake, baseline biomarker data, history of respiratory disease, hypertension, diabetes, and history of cardiovascular disease (e.g. including ischemic heart disease, heart failure, and stroke/TIA), as well as history of traumatic brain injury.

\section{Data analyses}

All analyses were conducted separately for each clinical group (i.e. NL, MCI, and AD). Cross-sectional analyses of baseline $\mathrm{AD}$ biomarker levels by OSA status were conducted using generalized linear methods.

Multivariate analysis of variance (MANOVA) was conducted to test differences in time-trend, groups (OSA+ vs. OSA-) and time points. To test differences in time-trend, baseline biomarker data were subtracted from data at each time point.

ADNI data are unbalanced with unequal numbers of measurement for each participant. We therefore used multilevel mixed effects linear regression models with normal errors [20], examining the relationship between OSA and the rate of change in AD biomarkers. This allowed the incorporation of all available information and possibly reduced bias resulting from using only the complete cases [21].

To determine the specific type of modeling, we examined profile plots for the biomarker levels over time by OSA status, using both the original dataset and within-subject residuals. We also examined trajectory plots obtained by subtracting the baseline measurement from the original measurements (i.e. $\mathrm{dij}=$ Yij - Yi1) and examined plots of mean and variance of the biomarkers at each time point by OSA status. Further, using the slopes as summary statistics, we conducted different formal tests to compare groups. Our assumption was that the rate of change of biomarker data is approximately linear over time. Thus, we used a parametric model with time as a continuous variable. We fitted the models with randomly varying intercepts and slopes and allowed them to depend on exposure group (i.e. OSA status). Based on the findings, we then fitted a random coefficients model to analyze the relationship between OSA status and time using unstructured covariance. To find a parsimonious covariance structure, we fitted many different covariance models and conducted a likelihood ratio test of each nested model using information criteria for non-nested models. Finally, using the unstructured, independence covariance model (i.e. the preferred covariance structure), we tested whether the rate of change in biomarkers differed between OSA groups. Final models were adjusted for age, sex, BMI, education, CPAP use, ApoE4 status, alcohol intake, baseline biomarker data, history of respiratory disease, hypertension, diabetes, and history of cardiovascular disease (e.g. including ischemic heart disease, heart failure, and stroke/TIA), and history of traumatic brain injury.

Adjustment for the extensive list of covariates had little impact on the estimates. The age and sex only-adjusted model and the extensively adjusted model showed similar results, indicating little to no risk of bias due to overadjustment. Sensitivity analysis removing CPAP users (NL: $n=10$; MCI: $n=16$; $\mathrm{AD}: n=9$ ) from OSA+ participants had also a negligible impact on the estimates (e.g. NL florbetapir estimate of .06 changed to .08). Statistical analyses were performed using SAS (version 9.4; SAS Institute Inc., Cary, NC).

These analyses allowed us to examine whether significant variation between OSA subjects in mean AD biomarker level at baseline (intercept) existed, as well as whether significant variation in the change in AD biomarker level over time (slope) occurred. Furthermore, the covariance between the baseline $\mathrm{AD}$ biomarker level (intercept) and $\mathrm{AD}$ biomarker change over time indicated whether OSA + or OSA-subjects had experienced a faster increase or decrease in $\mathrm{AD}$ biomarker level over time (significant slope). It also allowed for assessment of significant differences in the rate of change in $\mathrm{AD}$ biomarker level between OSA+ and OSA- patient groups over time.

\section{Data availability statement}

Authors state that anonymized data will be shared by request from any qualified investigator.

\section{Results}

\section{Demographic and clinical characteristics}

Figure 1 shows the stepwise exclusion process for NL, MCI, and AD participants used in the study. Table 1 shows the demographic and clinical characteristics of study participants at baseline. Overall, the mean ages of OSA+ and OSA- were $72.3 \pm 7.1$ and $73.9 \pm 7.3$ years, respectively. Female participants represented $49 \%, 40 \%$, and $37 \%$ of the NL, MCI, and AD groups, respectively. Six percent were OSA+ in the NL group, while $13 \%$ and $7 \%$ were $\mathrm{OSA}+$ in the $\mathrm{MCI}$ and $\mathrm{AD}$ groups. Participants differed markedly in ApoE4 status with 28\%,51\%, and 66\% being ApoE4 positive in 
the NL, MCI, and AD groups. NL, MCI, and AD participants were similar in age, education, BMI, and the three medical conditions across groups. Mean follow-up time was $2.52 \pm 0.51$ years.

\section{Baseline AD biomarker levels by clinical group and OSA status}

Table 1 and Supplementary Figure 1a-c show raw baseline CSF A $\beta 42$, T-tau, and P-tau levels, and florbetapir PET uptake in $\mathrm{NL}, \mathrm{MCI}$, and AD groups, by OSA status. There were significant differences in CSF $A \beta 42$ levels for both MCI ( $F=4.37, p=.04$ ), and $\mathrm{AD}(F=6.89, p<.01)$ groups, respectively (Figure 1$)$, with OSA+ participants having significantly higher levels at baseline for the MCI group. Significant difference in T-tau levels was seen for the MCI patients ( $F=5.08, p=.02$ ) (Figure 1$)$ with OSA+ individuals having significantly lower levels. These differences remained after controlling for covariates, but the magnitude of the differences was small. For florbetapir values, there were significant differences between OSA groups for both NL and MCI participants ( $F=5.53, F=5.15, p \leq .02$ for all, respectively) (Figure 1) with OSA+ participants having significantly lower florbetapir values. However, after controlling for age, sex, BMI, education, CPAP use, ApoE4 status, and other medical conditions, these differences in florbetapir values were no longer significant. For P-tau levels, no significant difference was seen across all groups in the uncontrolled and controlled analyses.

\section{Differences in time trend, groups (OSA+ vs. OSA-) and time-points}

Table 2, Figures 2-4, and Supplementary Figure 2a-c show MANOVA results testing the differences in the mean change in AD biomarker values over time, based on OSA status. The Pillai's trace test values presented examines time-point $\times$ OSA effect and provides the exact $F$ statistics for the time trend of mean change in AD biomarker across OSA groups. The time-point effect provides the exact $F$ statistics for the mean change (increase or decrease) in Alzheimer's disease biomarker over time. Other stats interpretations can be seen in the Table 2 footnote.

In NL and MCI participants, the time trend of mean change in florbetapir PET value indicated the change was not parallel across groups (Pillai's trace test, $p=.01$ for all). Across all subjects, mean change in florbetapir uptake increased significantly over time (Pillai's trace test, $p<.001$ for all). There were significant differences in mean change in florbetapir values across the OSA groups when the previous time-point was compared with the next $(p \leq .01$ for all).

In $\mathrm{AD}$ participants, the only significant finding was that the mean change in florbetapir uptake increased over time across all subjects (Pillai's trace test, $p<.001$ ).

In NL and MCI participants, the time trend of mean change in CSF A 42, T-tau, and P-tau values indicated the change was not parallel across groups (Pillai's trace test, $p \leq .05$ for all). Across all subjects, mean change in CSF A $\beta 42$ values decreased while T-tau and P-tau values increased over time (Pillai's trace test, $p<.001$ for all). There were significant differences in mean change in CSF $A \beta 42$, and T-tau values across the OSA groups when the previous time-point was compared with the next ( $p \leq .01$ for all). For P-tau, significant differences were only seen when timepoint_1 (year 2) was compared with timepoint_0 (year 1) $(p<.001)$.
In $\mathrm{AD}$ participants, the time trend of mean change in CSF T-tau indicated the change was not parallel across groups (Pillai's trace test, $p \leq .01)$. Across all subjects, mean change in CSF A $\beta 42$ values decreased while $P$-tau values increased significantly over time (Pillai's trace test, $p<.001$ for all). There were significant differences in mean change in T-tau values across the OSA groups only when timepoint_1 (year 2) was compared with timepoint_0 (year 1$)(p<.001)$. No significant differences were seen for CSF $A \beta 42$.

\section{Rate of change in AD biomarker by OSA status}

Table 3 reports the between-subjects variation and covariance parameter estimates in $\mathrm{AD}$ biomarker levels over time by OSA group.

In both NL and MCI participants at baseline, OSA+ participants had lower levels of CSF T-tau, CSF P-tau and brain florbetapir values and higher CSF A 342 levels, compared with OSAsubjects (Table 1). Interpretation of the covariance parameters is such that, if the signs of these estimates are negative, they show that persons with higher baseline values tend to have less rates of change over time. For example, in NL participants, for the flobertapir values, the covariance parameter estimate of -.06 $(-.09,-.04)$ indicates that OSA- participants who had a higher baseline tended to have slower rates of amyloid deposition over time (Figure 2). The covariance parameter estimate for CSF A $\beta 42$ of $3.93(3.56,4.31)$ indicates that OSA+ who had higher baseline values tended to have higher rates of changes over time (in this case decline because of the negative slope).

In NL participants, there was significant variation by OSA status in the annual change in brain florbetapir uptake (mean SUVR; $B=0.06, p<.0001$ ). In MCI participants, there was also significant variation by OSA status in the annual change in florbetapir values (mean SUVR; $B=0.08, p<.0001$ ). The covariance between the baseline florbetapir uptake and the annual florbetapir value change was -.06 , indicating that OSA+ subjects experienced faster increase in amyloid deposition over time $(p<.0001)$ when compared with OSA- (Figure 3 and Supplementary Figure $2 b$ ). No significant variation in the change in brain florbetapir PET volumes by OSA status was seen in the AD group (Table 3 and Figure 4).

For the NL and MCI groups, there was significant variation in the annual change in CSF A 42 by OSA status (mean CSF levels; $B=-2.708$, mean CSF levels; $B=-2.264, p<.0001$ for all, respectively). Significant differences in the annual change in T-tau and P-tau levels over time were also observed in both groups. Covariance parameters between the baseline CSF A 442 , T-tau, and P-tau level change over time indicated that OSA+ subjects experienced a faster longitudinal decrease in CSF A 442 and faster longitudinal increases in T-tau and P-tau levels $(p<0.0001$ for all) compared with OSA- participants, in both the NL and MCI groups (Table 3 and Figures 2-4). No significant variations in the change in CSF A $342, \mathrm{~T}$-tau, and P-tau levels over time were seen for the AD group (Table 3).

\section{Discussion}

The major objective of this study was to examine the association of OSA and longitudinal changes in brain amyloid PET deposition and CSF biomarkers. There were significant differences in the 


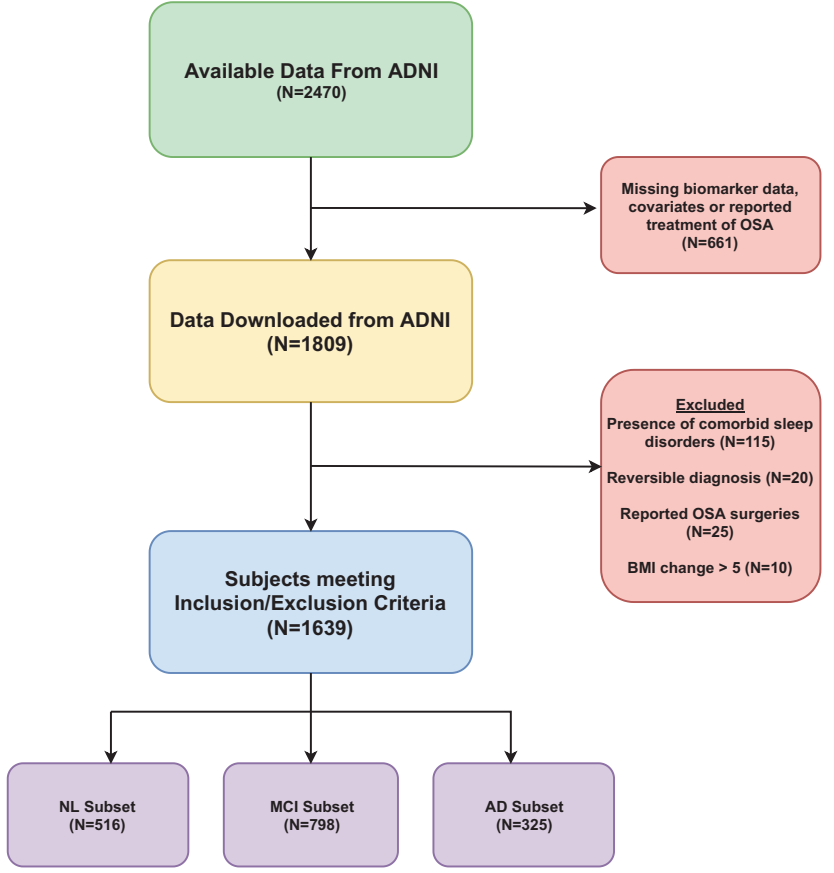

Figure 1. Stepwise exclusion process for NL, MCI, and AD participants used in the study.

annual rate of change in florbetapir uptake as well as CSF A $\beta 42$, T-tau, and P-tau levels over the follow-up period for the NL and MCI groups, with OSA+ subjects experiencing significantly faster increase in brain amyloid load (as measured by increases in florbetapir uptake and decreases in CSF A 342 levels) and tau aggregates (as measured by increases in CSF T-tau and P-tau levels). The direction of these longitudinal changes is in accordance with growing evidence described below showing a link between disturbed sleep, OSA, and AD pathogenesis. In a cross-sectional study, Spira et al. [22] found that MCI individuals with higher apnea-hypopnea and oxygen desaturation indices demonstrated higher amyloid deposition on Pittsburgh compound B (PiB)-PET uptake globally and regionally in the precuneus. Recently, Yun et al. [7] found that OSA patients had a higher amyloid burden in the right posterior cingulate gyrus and right temporal cortex relative to controls, suggesting possible contribution of OSA. In a population of NL individuals, Liguori et al. [5] compared CSF biomarkers in OSA+ versus OSA+ treated with CPAP as well as OSA- controls. Findings demonstrated lower CSF A 342 and higher T-tau/A $\beta 42$ ratio in OSA+ compared with CPAP treated and OSA- control subjects. We also have recently documented that OSA severity is associated with increased amyloid burden (measured as longitudinal decreases in CSF A 342 and increases in PiB uptake) over a 2-year follow-up in community-dwelling NL elderly [23].

Possible mechanisms responsible for these findings include intermittent hypoxia, sleep fragmentation, and intrathoracic pressure swings (Figure 5). Studies of cerebral ischemia suggest that both acute and intermittent hypoxia may promote $A \beta$ accumulation [2, 4, 24-26], possibly mediated by hypertension [27], cardiovascular disease [28], diabetes [29], chronic inflammation [30], and oxidative stress [31, 32]. Sleep fragmentation, arousals, and circadian rhythm disruption have also been shown to increase the risk of developing MCI/AD [1,
33], possibly through disruption of slow wave sleep (SWS) [34, 35]. Findings from a recent prospective study on subjects from the Framingham Heart Study cohort; suggest that OSA-related REM disruption may also contribute to this association [36]. Other possible mechanisms include elevated intrathoracic, intracranial pressures, and/or increased venous pressure, which have been hypothesized to acutely and repetitively impede the circulation of brain metabolites from the interstitial fluid (ISF) into the CSF through the glymphatic system, leading to increases in $A \beta$ accumulation [24, 31, 32, 37]. Lastly, depression has been reported as both a consequence of OSA and an AD risk factor and may also contribute to this association [38].

Intriguingly, at baseline, our data showed that OSA+ patients had significantly higher CSF A 442 in MCIs, lower florbetapir uptake in both NL and MCIs, and lower T-tau levels in MCIs, compared with OSA- subjects. A possible concern here is a regression toward the mean, since the OSA+ group starts with significantly lower values of amyloid load in some of the biomarkers used. However, there are two factors worth addressing in this regard. First, the absolute difference in these levels between groups was numerically quite small (e.g. in NL subjects, the difference in the median CSF A 442 levels was 17 $\mathrm{pg} / \mathrm{mL}$ ). Second, the longitudinal analyses examined the "rate of change" as an outcome and not the mean levels of the AD biomarkers. The longitudinal mean beta statistics presented refer to the mean change incorporating within and between group data. In addition, what we examined was the slope statistics incorporating the effects of time, which looked at the rate of change for each individual between OSA groups. Furthermore, one could expect faster progression of amyloid deposition in the NL group that has already higher amyloid burden at baseline $[39,40]$, which would have driven our findings toward the null. Possible explanations for the reduced baseline $\mathrm{AD}$ biomarker presentation in the OSA+ group include; first, if OSA accelerates AD pathology buildup this may simply be a sign of selection (survival) bias as most OSA+ will have transitioned to MCI or AD and only those with very low $\mathrm{AD}$ pathology burden at disease onset would remain as cognitively normal. Second, particularly in those with MCI, is the presence of increased vascular risk in this group (i.e. higher rates of diabetes and obesity) (Table 1), as onset of cognitive impairment is likely to occur at a lower severity of Alzheimer lesions in individuals with more vascular pathology [41]. Notably, after age stratification (55-73.8, and 74-92) based on age distribution (we categorized based on the 50th percentile), compared with OSA- individuals, OSA+ individuals in the 55-73.8 years old category were more likely to have higher vascular burden (i.e. composite score for BMI, hypertension, diabetes, and cardiovascular disease), higher CSF A 42 in MCIs, lower florbetapir uptake in both NL and MCIs, and lower T-tau levels in MCIs (Supplementary Table 1). In addition, among ages 74 years and above, OSA+ participants had a worse $\mathrm{AD}$ biomarker presentation (i.e. lower CSF A $\beta 42$ in MCIs, higher florbetapir uptake in both NL and MCIs, and higher T-tau levels in MCIs) at baseline. These findings suggest that the younger population especially in the OSA+ with higher vascular burden were driving the noted differences at baseline between OSA+ versus OSA-groups. Third, specifically for CSF A $\beta 42$; slow wave sleep (SWS) occurring during nonrapid eye movement (NREM) sleep, a known A $\beta$ modulator [35, 42, 43], may be responsible. OSA is associated with reduced SWS [44]. Sleep is involved in the clearance of $A \beta$ [35], and fragmented SWS limits brain $A \beta$ 
Table 1. Descriptive characteristics of participants by self-reported obstructive sleep apnea status at baseline

\begin{tabular}{|c|c|c|c|}
\hline Characteristics & All & OSA- & OSA+ \\
\hline \multicolumn{4}{|c|}{ COGNITIVE NORMAL } \\
\hline Number of participants (\%) & $516(100)$ & $487(94)$ & $29(6)$ \\
\hline Female sex, number (\%) & $253(49)$ & $244(50)$ & $9(32)$ \\
\hline Age, years, median (interquartile range) & $74(71,78)$ & $71(70,78)$ & $71(70,76)$ \\
\hline ApoE4 positive, number (\%) & $145(28)$ & $140(29)$ & $5(17)$ \\
\hline Education, years, median (interquartile range) & $16(14,18)$ & $16(14,18)$ & $16(15,18)$ \\
\hline BMI $\left(\mathrm{kg} / \mathrm{m}^{2}\right)$ & $27.2 \pm 4.8$ & $27.1 \pm 4.7$ & $29.8 \pm 5.9$ \\
\hline Hypertension, number (\%) & $251(49)$ & $235(48)$ & $16(55)$ \\
\hline Diabetes, number $(\%)$ & $45(9)$ & $40(8)$ & $5(17)$ \\
\hline Thyroid disease, number (\%) & $112(22)$ & $109(22)$ & $3(10)$ \\
\hline Respiratory disease, number (\%) & $123(24)$ & $100(21)$ & $23(79)$ \\
\hline Cardiovascular disease, number (\%) & $358(69)$ & $338(69)$ & $20(69)$ \\
\hline TBI, number (\%) & $13(3)$ & $12(3)$ & $1(3)$ \\
\hline Alcohol, number (\%) & $23(5)$ & $22(5)$ & $1(3)$ \\
\hline CPAP use & $10(19)$ & & $10(35)$ \\
\hline CSF amyloid beta $42 \mathrm{pg} / \mathrm{mL}$ median (interquartile range) & $210(155,241)$ & $209(155,241)$ & $226(199,259)$ \\
\hline T-tau pg/mL median (interquartile range) & $59(45,84)$ & $59(4583)$ & $56(48,84)$ \\
\hline P-tau pg/mL median (interquartile range) & $27(20,40)$ & $27(20,42)$ & $27(20,32)$ \\
\hline Florbetapir SUVR, median (interquartile range)* & $1.1(1.0,1.2)$ & $1.1(1.0,1.2)$ & $1.0(1.0,1.0)$ \\
\hline MMSE median (interquartile range) & $29(28,29)$ & $29(28,30)$ & $29(27,30)$ \\
\hline CDR median (interquartile range) & $0(0,0)$ & $0(0,0)$ & $0(0,0)$ \\
\hline \multicolumn{4}{|c|}{ MILD COGNITIVE IMPAIRMENT } \\
\hline Number of participants (\%) & $798(100)$ & $695(87)$ & $103(13)$ \\
\hline Female sex, number $(\%)$ & $319(40)$ & $294(42)$ & $25(25)$ \\
\hline Age, years, median (interquartile range) & $74(68,79)$ & $71(70,78)$ & $71(70,76)$ \\
\hline ApoE4 positive, number (\%) & $410(51)$ & $368(53)$ & $42(41)$ \\
\hline Education, years, median (interquartile range) & $16(14,18)$ & $16(14,18)$ & $16(14,18)$ \\
\hline BMI $\left(\mathrm{kg} / \mathrm{m}^{2}\right)$ & $26.9 \pm 4.6$ & $26.5 \pm 4.4$ & $29.4 \pm 5.3$ \\
\hline Hypertension, number (\%) & $395(49)$ & $337(48)$ & $58(56)$ \\
\hline Diabetes, number (\%) & $75(9)$ & $57(8)$ & $18(17)$ \\
\hline Thyroid disease, number (\%) & $156(20)$ & $133(19)$ & $23(22)$ \\
\hline Respiratory disease, number (\%) & $195(24)$ & $133(19)$ & $62(60)$ \\
\hline Cardiovascular disease, number (\%) & $577(72)$ & $490(71)$ & $87(85)$ \\
\hline TBI, number (\%) & $18(2)$ & $14(2)$ & $4(4)$ \\
\hline Alcohol, number (\%) & $35(4)$ & $31(5)$ & $4(4)$ \\
\hline CPAP use & $16(20)$ & & $16(16)$ \\
\hline CSF amyloid beta $42 \mathrm{pg} / \mathrm{mL}$ median (interquartile range)* & $153(130,209)$ & $150(128,206)$ & $169(139,214)$ \\
\hline T-tau pg/mL median (interquartile range)* & $80(54,116)$ & $81(54,122)$ & $67(51,97)$ \\
\hline P-tau pg/mL median (interquartile range) & $36(23,51)$ & $37(23,52)$ & $32(21,44)$ \\
\hline Florbetapir SUVR, median (interquartile range)* & $1.2(1.0,1.4)$ & $1.2(1.0,1.4)$ & $1.1(1.0,1.3)$ \\
\hline MMSE median (interquartile range) & $27(24,28)$ & $28(25,29)$ & $26(25,28)$ \\
\hline CDR median (interquartile range) & $0.5(0.5,0.5)$ & $0.5(0.5,0.5)$ & $0.5(0.5,0.5)$ \\
\hline \multicolumn{4}{|c|}{ ALZHEIMER'S DISEASE } \\
\hline Number of participants (\%) & $325(100)$ & $303(93)$ & $22(7)$ \\
\hline Female sex, number (\%) & $119(37)$ & $113(37)$ & $6(27)$ \\
\hline Age, years, median (interquartile range) & $76(71,80)$ & $76(71,80)$ & $71(64,76)$ \\
\hline ApoE4 positive, number (\%) & $216(66)$ & $198(65)$ & $18(82)$ \\
\hline Education, years, median (interquartile range) & $16(14,18)$ & $16(14,18)$ & $16(14,18)$ \\
\hline BMI $\left(\mathrm{kg} / \mathrm{m}^{2}\right)$ & $25.9 \pm 4.6$ & $25.6 \pm 4.3$ & $29.0 \pm 6.8$ \\
\hline Hypertension, number (\%) & $165(51)$ & $149(49)$ & $16(73)$ \\
\hline Diabetes, number (\%) & $32(10)$ & $30(10)$ & $2(9)$ \\
\hline Thyroid disease, number (\%) & $65(20)$ & $61(20)$ & $4(18)$ \\
\hline Respiratory disease, number (\%) & $67(21)$ & $52(17)$ & $15(68)$ \\
\hline Cardiovascular disease, number (\%) & $222(68)$ & $203(67)$ & $19(86)$ \\
\hline TBI, number (\%) & $18(2)$ & $14(2)$ & $4(4)$ \\
\hline Alcohol, number (\%) & $20(6)$ & $17(6)$ & $3(14)$ \\
\hline CPAP use & $9(3)$ & & $9(41)$ \\
\hline CSF amyloid beta $42 \mathrm{pg} / \mathrm{mL}$ median (interquartile range)* & $132(116,151)$ & $115(96,182)$ & $113(79,151)$ \\
\hline T-tau pg/mL median (interquartile range) & $80(54,116)$ & $67(51,97)$ & $81(54,122)$ \\
\hline P-tau pg/mL median (interquartile range) & $42(33,61)$ & $41(33,61)$ & $54(35,66)$ \\
\hline Florbetapir SUVR, median (interquartile range) & $1.4(1.3,1.5)$ & $1.4(1.3,1.5)$ & $1.4(1.3,1.6)$ \\
\hline MMSE median (interquartile range) & $24(20,26)$ & $24(22,26)$ & $24(20,25)$ \\
\hline CDR median (interquartile range) & $2.0(1.0,2.5)$ & $1.5(1.0,2.5)$ & $2.0(1.0,2.5)$ \\
\hline
\end{tabular}

$\mathrm{A} \beta=$ amyloid beta; $\mathrm{ApoE} 4$ = apolipoprotein epsilon4; $\mathrm{BMI}=$ body mass index; $\mathrm{CDR}=$ clinical dementia rating; $\mathrm{CPAP}=$ continuous pulmonary airway pressure; $\mathrm{CSF}$ = cerebrospinal fluid; MMSE = Mini-Mental State Exam; tau = tau protein; P-tau = phosphorylated tau; TBI = traumatic brain injury. ${ }^{*}$ Significant differences between groups (CSF A 342 levels for both $\mathrm{MCI}[\mathrm{F}=4.37, p=.04]$, and $\mathrm{AD}[\mathrm{F}=6.89, p<.01]$ and $\mathrm{T}$-tau levels for the MCI patients $[\mathrm{F}=5.08, p=.02]$ ). 


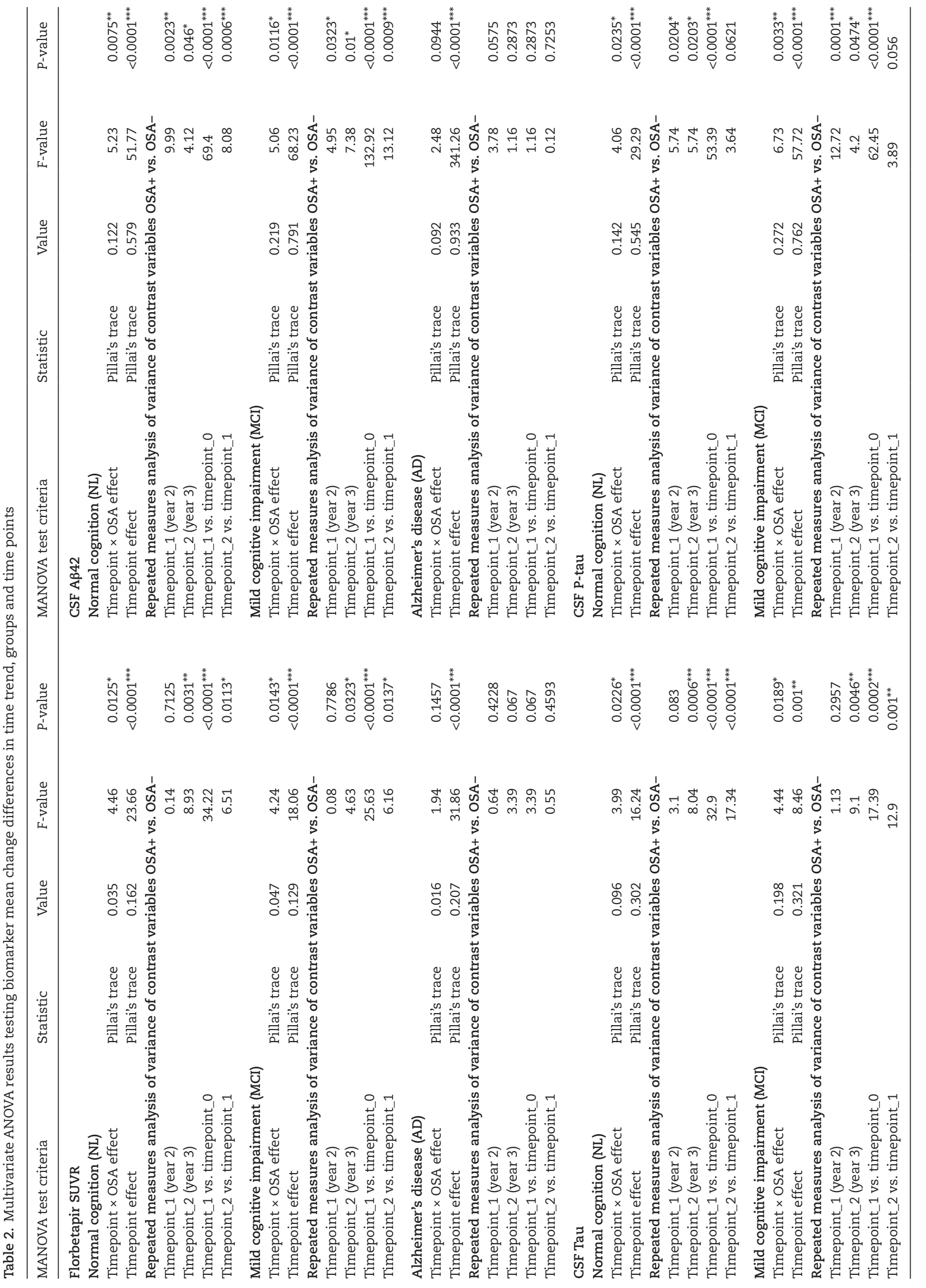




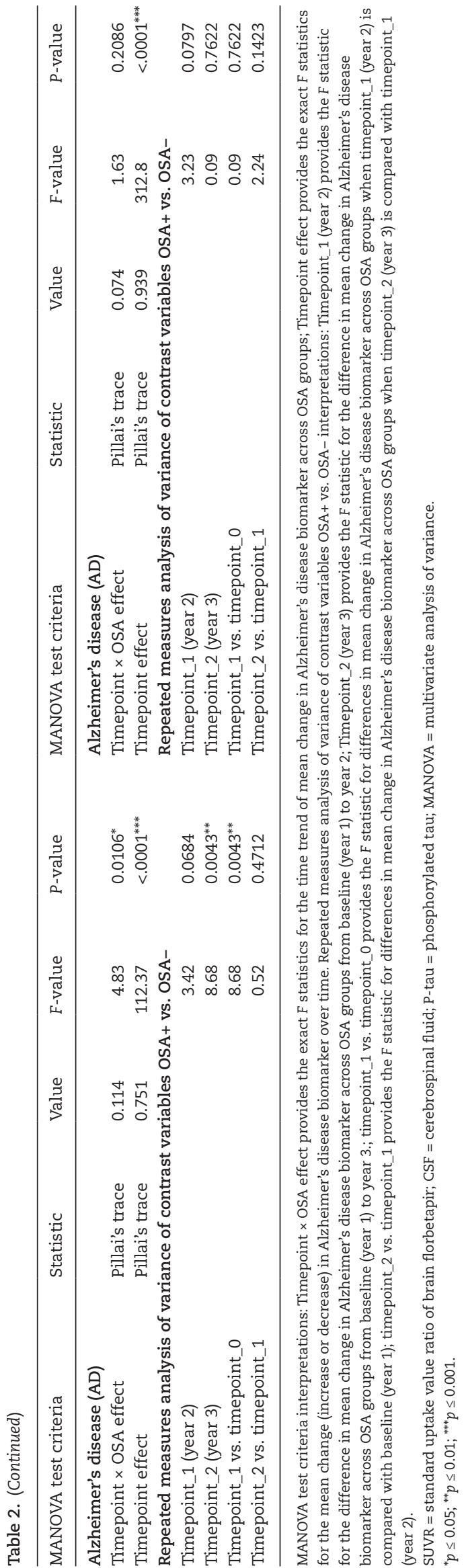

clearance, thereby leaving higher levels that reflect in the CSF $[45,46]$. At cross-section, disrupted SWS has been shown to be associated with higher CSF A $\beta 42$ in middle-aged [24, 47] and older adults [34]. Fourth, in both the NL and MCI groups, OSAhad more APOE4 positive participants, which is known to confer higher risk and worse $\mathrm{AD}$ biomarker burden [48]. It is important to note that as stated above (see Baseline AD biomarker levels by clinical group and OSA status under Results section), after controlling for age, sex, BMI, education, CPAP use, ApoE4 status, and other medical conditions, the differences in florbetapir values were no longer significant. For P-tau levels, no significant difference was seen across all groups in the uncontrolled and controlled analyses. In other words, these differences are numerically but not statistically different from each other, and where they were "statistically" significantly different, it is not clear that they are clinically meaningfully different.

Our findings in a cohort of self-reported OSA patients suggest that untreated OSA can predispose to neurodegenerative processes in the long-term by gradually weakening brain structure and altering its functioning $[49,50]$. This hypothesis would be consistent with an overarching model of lateonset $\mathrm{AD}$ with brain amyloid deposition and tau aggregates proceeding at different rates, influenced by a combination of protective/risk factors of which OSA is part [51]. This model of AD implies a contributory role of OSA severity in affecting and/ or accelerating $\mathrm{AD}$ biomarker change and possibly increasing neurodegeneration. In agreement with these findings, our previous study performed in the ADNI cohort showed that the presence of OSA was associated with accelerated cognitive decline and an earlier age of MCI or AD dementia onset [52]. As expected, there was no difference in the rate of biomarker change in the $\mathrm{AD}$ group by OSA status. This may be because in $\mathrm{AD}$ patients, brain amyloid is exhibiting a ceiling effect at higher levels of brain atrophy [53]. OSA's effect as it relates to $\mathrm{AD}$ biomarker changes may therefore be attenuated after it has reached its maximum neuronal damage.

\section{Strengths and limitations}

Our study possesses several strengths including a well-defined cohort, and objective assessment of amyloid burden and CSF P-tau/T-tau, which allowed for a high degree of certainty regarding measurement of clinical groups and outcomes [54]. Our statistical analytic methods were also robust with respect to unbalanced number of observations per subject over time.

We acknowledge we were limited by the measurement of OSA by self-report. Self-reported sleep measures can be impacted by diminished cognition [55] and in certain situations might not be correlated with objective methods [56]. The prevalence of reported OSA was also significantly lower than expected in this elderly cohort, which can relate to an underdiagnosis effect in this population, as epidemiological and sleep laboratory studies document much higher OSA prevalence in elderly populations. Therefore, some OSA+ subjects were likely misclassified into the OSA- group; however, this would have driven our findings toward the null. However, one implication of OSA classification by self-report is that those with self-reported OSA very likely had associated symptoms (i.e. excessive daytime sleepiness) that prompted these subjects to seek diagnosis. The overall prevalence of self-reported OSA in this group (6\%) is similar to the US prevalence of the OSA syndrome (OSAS) (4\%) defined by the 


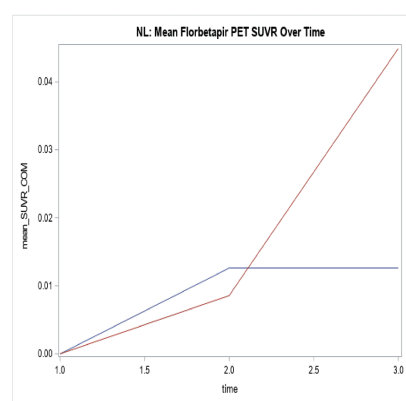

OSA

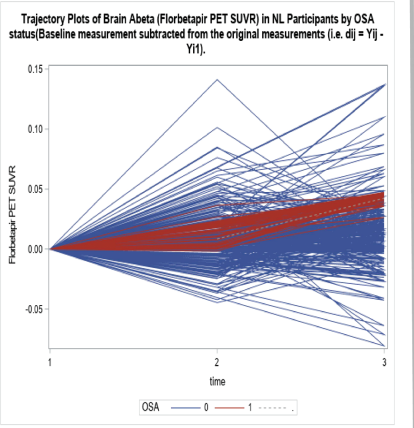

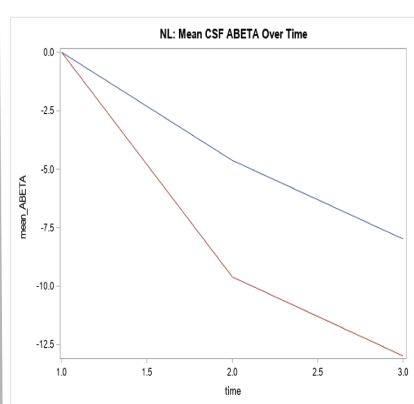

OSA $-0-1$

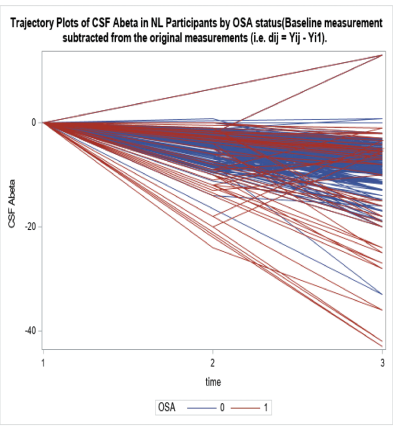

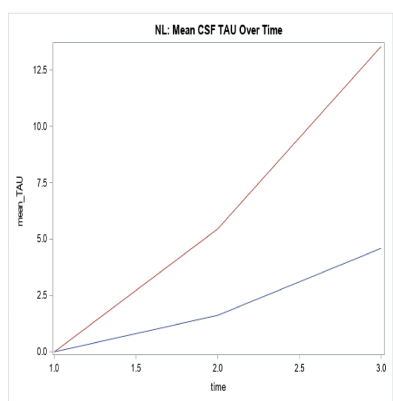

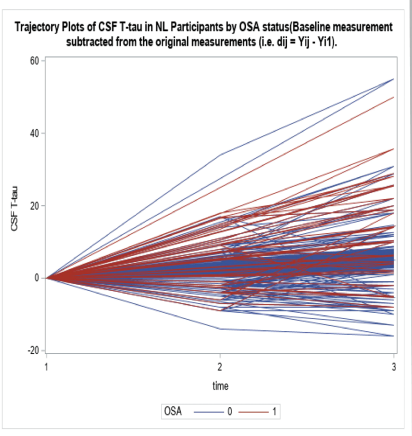

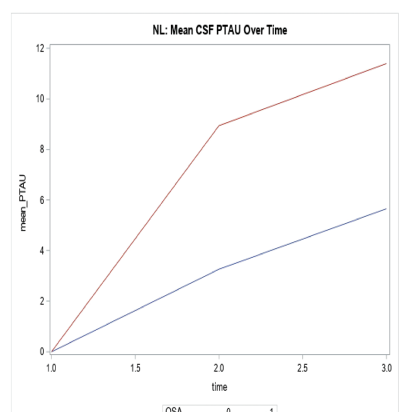

OSA -
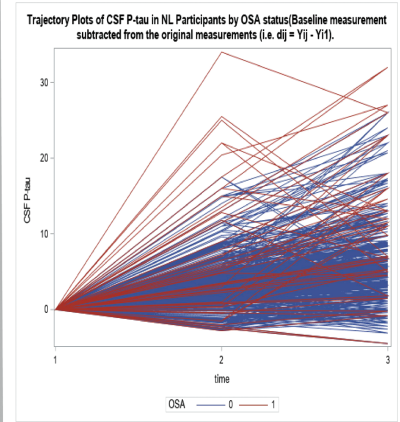

Figure 2. Mean change variations and trajectory plots in AD biomarker values over time in NL, MCI, and AD subjects by OSA status (cognitive normal-NL).
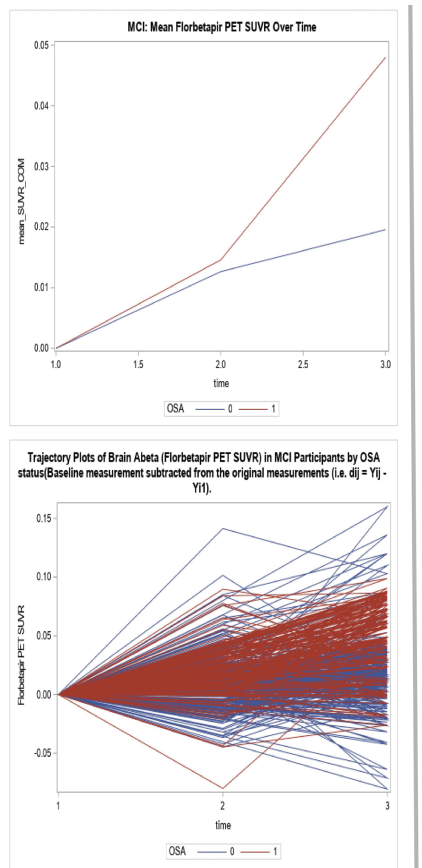

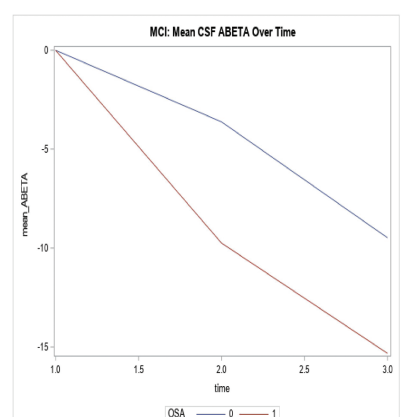

OSA $-0-1$

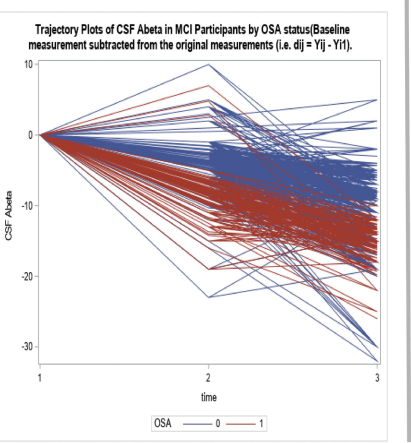

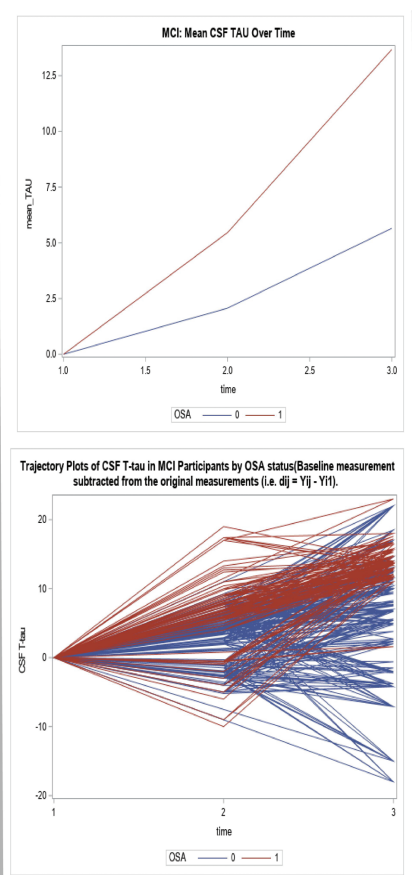
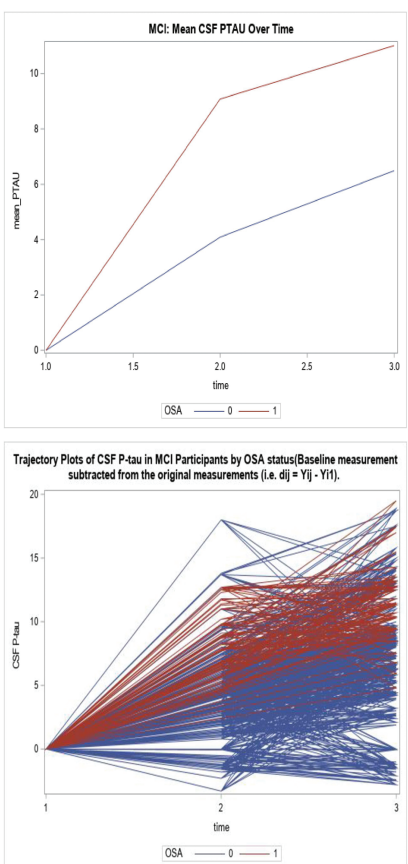

Figure 3. Mean change variations and trajectory plots in AD biomarker values over time in NL, MCI, and AD subjects by OSA status (mild cognitive impairment-MCI).

presence of $\mathrm{AHI} 4 \% \geq 5 / \mathrm{h}$ and daytime symptoms across all ages [57]. The prevalence of OSA (with or without symptoms) in the elderly is much higher, estimated at $30 \%-50 \%$ in older subjects [58], thus additional work may be required to differentiate the risk of OSA for $\mathrm{AD}$ with and without associated daytime symptoms. Notably, all cause excessive daytime sleepiness in elderly subjects defined by Epworth sleepiness scores $\geq 10$ was associated with longitudinal brain beta amyloid accumulation in a recent study [59].

\section{Conclusions}

OSA appears to accelerate increases in amyloid deposition, CSF T-tau and P-tau levels over time, both in NL and MCI individuals. Sleep fragmentation, intermittent hypoxia, and intrathoracic pressure swings from OSA are likely candidate mechanisms. Thus, clinical interventions aimed at OSA, such as treatment with CPAP or dental appliances, in cognitive normal and MCI 

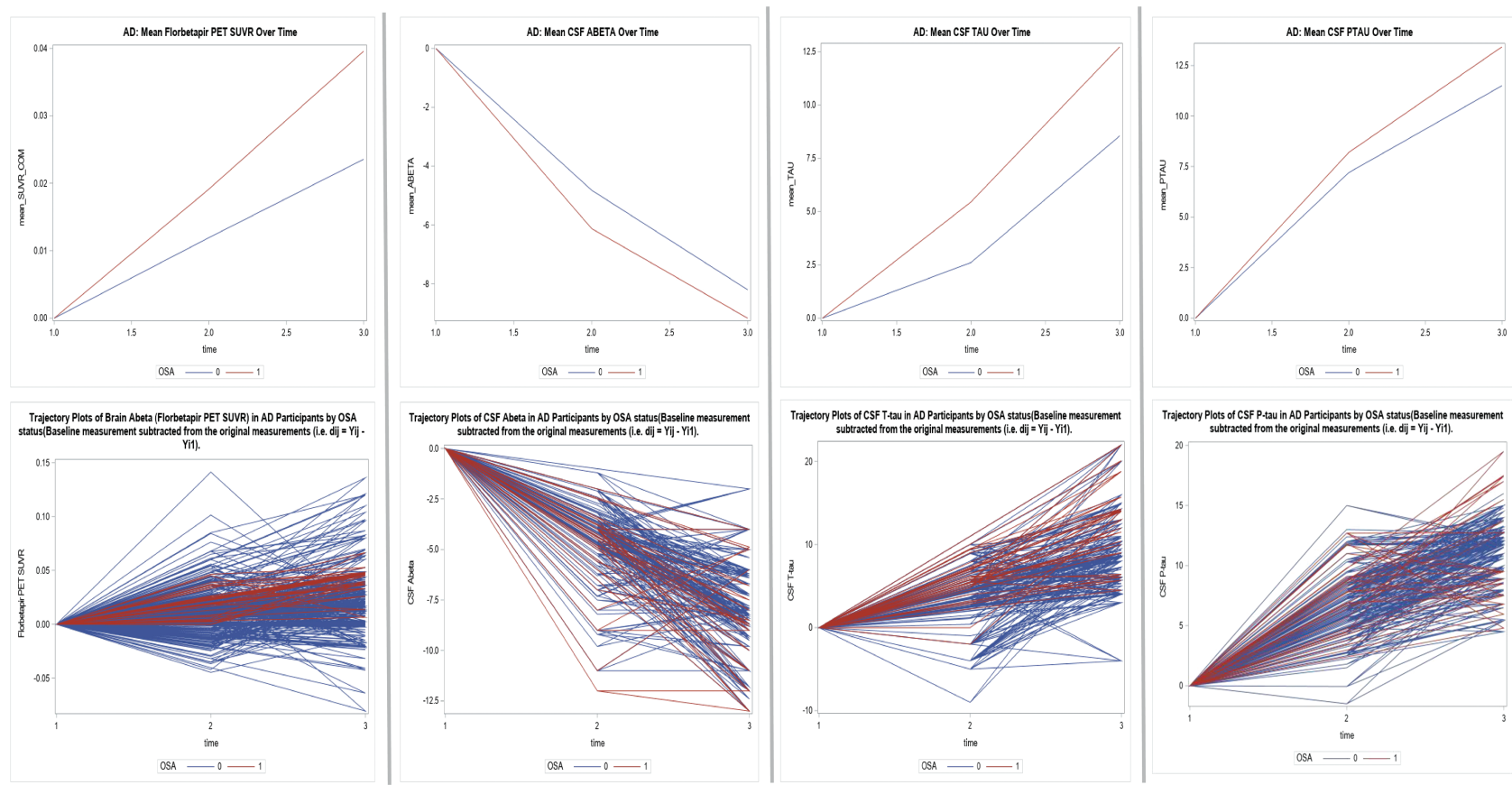

Figure 4. Mean change variations and trajectory plots in AD biomarker values over time in NL, MCI, and AD subjects by OSA status (Alzheimer's disease-AD).

Table 3. Between subject variation in $\mathrm{AD}$ biomarkers and covariance parameter estimates

\begin{tabular}{|c|c|c|c|}
\hline Parameters & Estimate & $95 \%$ CI & $P$-value \\
\hline \multicolumn{4}{|c|}{ OSA+ vs. OSA- (cognitive normal patients) } \\
\hline Florbetapir SUVR over time & 0.06 & $.02, .11$ & $<0.0001$ \\
\hline Florbetapir SUVR over time (covariance) & -0.06 & $-.09,-.04$ & $<0.0001$ \\
\hline CSF $A \beta-42$ over time & -2.71 & $-3.11,-2.35$ & $<0.0001$ \\
\hline CSF A $\beta$-42 over time (covariance) & 3.93 & $3.56,4.31$ & $<0.0001$ \\
\hline CSF T-tau over time & 3.68 & $3.31,4.07$ & $<0.0001$ \\
\hline CSF T-tau over time (covariance) & -2.89 & $-3.51,-2.29$ & $<0.0001$ \\
\hline CSF P-tau over time & 1.22 & $1.02,1.42$ & $<0.0001$ \\
\hline CSF P-tau over time (covariance) & -1.21 & $-1.71,-0.74$ & $<0.0001$ \\
\hline \multicolumn{4}{|c|}{ OSA+ vs. OSA- (mild cognitive impairment patients) } \\
\hline Florbetapir SUVR over time & 0.08 & $.05, .12$ & $<0.0001$ \\
\hline Florbetapir SUVR over time (covariance) & -0.06 & $-0.09,-0.04$ & $<0.0001$ \\
\hline CSF A $\beta-42$ over time & -2.62 & $-3.23,-2.03$ & $<0.0001$ \\
\hline CSF A $\beta-42$ over time (covariance) & 2.69 & $2.02,3.36$ & $<0.0001$ \\
\hline CSF tau volume over time & 2.21 & $1.58,2.86$ & $<0.0001$ \\
\hline CSF tau over time (covariance) & -1.89 & $-2.91,-0.87$ & $<0.0001$ \\
\hline CSF P-tau over time & 1.74 & $1.22,2.27$ & $<0.0001$ \\
\hline CSF P-tau over time (covariance) & -1.48 & $-2.05,-0.94$ & $<0.0001$ \\
\hline \multicolumn{4}{|c|}{ OSA+ vs. OSA- (Alzheimer's disease patients) } \\
\hline Florbetapir SUVR over time & 0.07 & $-1.19,1.33$ & 0.33 \\
\hline Florbetapir SUVR over time (covariance) & -0.29 & $-2.07,1.49$ & 0.31 \\
\hline CSF A $\beta-42$ over time & -1.11 & $-3.31,1.09$ & 0.53 \\
\hline CSF A $\beta$-42 over time (covariance) & -1.14 & $-3.38,1.63$ & 0.56 \\
\hline CSF T-tau over time & 0.26 & $-1.02,1,28$ & 0.47 \\
\hline CSF T-tau over time (covariance) & -0.15 & $-1.94,1.64$ & 0.47 \\
\hline CSF P-tau over time & 0.94 & $0.23,1.65$ & 0.11 \\
\hline CSF P-tau over time (covariance) & -0.16 & $-1.66,1.34$ & 0.11 \\
\hline
\end{tabular}

These analyses allowed us to examine whether there was significant variation between OSA+ and OSA- subjects in mean AD biomarker level at baseline, as well as whether significant variation in the change in $\mathrm{AD}$ biomarker level over time occurred. Furthermore, the covariance between the baseline $\mathrm{AD}$ biomarker level and AD biomarker change over time indicated whether OSA+ or OSA- subjects had experienced a faster increase or decrease in AD biomarker level over time. It also allowed for assessment of significant differences in the rate-of-change in AD biomarker level between OSA groups over time. Models were adjusted for age, sex, BMI, education, CPAP use, ApoE4 status, alcohol intake, baseline biomarker data, history of respiratory disease, hypertension, diabetes, and history of cardiovascular disease (e.g. including ischemic heart disease, heart failure, and stroke/TIA), and history of traumatic brain injury. $\mathrm{A} \beta=$ amyloid beta; $\mathrm{ApoE4}=$ apolipoprotein epsilon4; $\mathrm{BMI}=$ body mass index; $\mathrm{CSF}=$ cerebrospinal fluid; tau = tau protein; $\mathrm{P}$-tau = phosphorylated tau . 


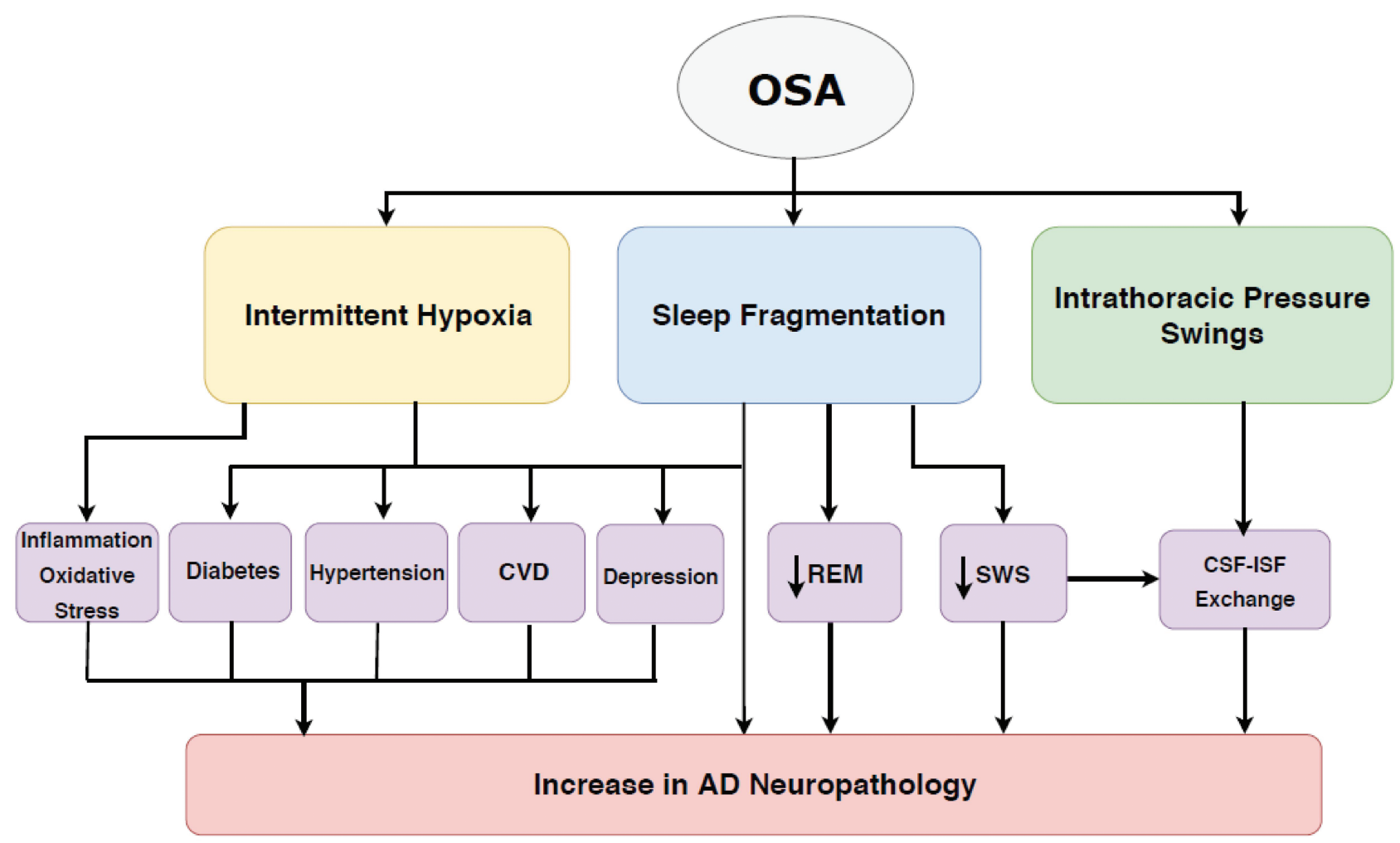

Figure 5. Proposed mechanisms linking OSA with amyloid deposition in late-life.

patients, could possibly mitigate or slow the progression of cognitive impairment to AD. Further studies examining the mechanisms underlying these observed effects are needed.

\section{Supplementary material}

Supplementary material is available at SLEEP online.

\section{Acknowledgments}

The authors would like to gratefully acknowledge the dedication, time, and commitment of the participants and staff of the Alzheimer's Disease Neuroimaging Initiative (ADNI). ADNI data are disseminated by the Laboratory for Neuro Imaging at the University of Southern California. Data used in preparation of this article were obtained from the Alzheimer's Disease Neuroimaging Initiative (ADNI) database (adni.loni.usc.edu). The investigators within the ADNI contributed to the design and implementation of ADNI projects and provided data, but did not participate in the design, analysis, or writing of this report. A complete listing of ADNI investigators can be found at: http:// adni.loni.usc.edu/wp content/uploads/how_to_apply/ADNI_ Acknowledgement_List.pdf. The authors would like to thanks Ms. Jatnna Rivas and Eirene Oji for their author contributions help with the figure.

\section{Authors Contributions}

O.M.B., R.S.O., E.P., A.A., and R.A.S. were involved in the conception and design of the study, acquisition and analysis of the data, as well as drafting a significant portion of the article or figures. S.G., O.Q.U., M.M.H., A.M.S.,
F.M., N.S., and A.K.M. were involved in the literature review, conception, and design of the study, and revision of the article for intellectual content. A.A.S., K.K., F.Z., A.R.B., J.A.M., K.E.K., D.M., I.R., I.A., D.M.R., G.J., and A.W.V. interpreted the data; revised the article for intellectual content and drafted significant portions of the article.

\section{Funding}

This work was supported by grants from the NIH/NIA/NHLBI (T32HL129953, R01HL118624, R21AG049348, R21AG055002, R01AG056031, R01AG022374, R21AG059179, R01AG056682, P30AG008051 and R01AG056531; Foundation for Research in Sleep Disorders; American Sleep Medicine Foundation, the American Thoracic Society Foundation, the Friedman Brain Institute. The Wellcome Trust (103952/Z/14/Z) supports I.R. Collection and sharing of data for this project was funded by ADNI (National Institutes of Health Grant U01 AG024904) and DOD ADNI (Department of Defense award number W81XWH-12-2-0012). ADNI is funded by the National Institute on Aging, the National Institute of Biomedical Imaging and Bioengineering, and through generous contributions from the following: AbbVie, Alzheimer's Association; Alzheimer's Drug Discovery Foundation; Araclon Biotech; BioClinica, Inc.; Biogen; Bristol-Myers Squibb Company; CereSpir, Inc.; Cogstate; Eisai, Inc.; Elan Pharmaceuticals, Inc.; Eli Lilly and Company; EuroImmun; F. Hoffmann-La Roche Ltd and its affiliated company Genentech, Inc.; Fujirebio; GE Healthcare; IXICO Ltd.; Janssen Alzheimer Immunotherapy Research \& Development, LLC.; Johnson \& Johnson Pharmaceutical Research \& Development LLC.; Lumosity; Lundbeck; Merck \& Co., Inc.; Meso Scale Diagnostics, LLC.; NeuroRx Research; Neurotrack Technologies; Novartis Pharmaceuticals Corporation; Pfizer, 
Inc.; Piramal Imaging; Servier; Takeda Pharmaceutical Company; and Transition Therapeutics. The Canadian Institutes of Health Research is providing funds to support ADNI clinical sites in Canada. Private sector contributions are facilitated by the Foundation for the National Institutes of Health (www.fnih.org). The grantee organization is the Northern California Institute for Research and Education, and the study is coordinated by the Alzheimer's Therapeutic Research Institute at the University of Southern California.

Conflict of interest statement. R.S.O. gave a paid talk to Eisai Global at the SLEEP 2017 meeting.

\section{References}

1. Lim AS, et al. Sleep fragmentation and the risk of incident Alzheimer's disease and cognitive decline in older persons. Sleep. 2013;36(7):1027-1032.

2. Nalivaevaa NN, et al. Effect of hypoxia/ischemia and hypoxic preconditioning/reperfusion on expression of some amyloid-degrading enzymes. Ann N Y Acad Sci. 2004;1035:21-33.

3. Polsek D, et al. Obstructive sleep apnoea and Alzheimer's disease: in search of shared pathomechanisms. Neurosci Biobehav Rev. 2018;86:142-149.

4. Sun $\mathrm{X}$, et al. Hypoxia facilitates Alzheimer's disease pathogenesis by up-regulating BACE1 gene expression. Proc Natl Acad Sci USA. 2006;103(49):18727-18732.

5. Liguori C, et al. Obstructive sleep apnea is associated with early but possibly modifiable Alzheimer's disease biomarkers changes. Sleep. 2017;40(5).

6. Spira AP, et al. Self-reported sleep and $\beta$-amyloid deposition in community-dwelling older adults. JAMA Neurol. 2013;70(12):1537-1543.

7. Yun $\mathrm{CH}$, et al. Amyloid burden in obstructive sleep apnea. J Alzheimers Dis. 2017;59(1):21-29.

8. Brys $\mathrm{M}$, et al. Prediction and longitudinal study of CSF biomarkers in mild cognitive impairment. Neurobiol Aging. 2009;30(5):682-690.

9. Waragai $\mathrm{M}$, et al. Comparison study of amyloid PET and voxel-based morphometry analysis in mild cognitive impairment and Alzheimer's disease. J Neurol Sci. 2009;285(1-2):100-108.

10. Buerger $\mathrm{K} \mathrm{HH}$. Evaluation of phosporylated tau protein as a core biomarker of Alzheimer's disease. In: Iqbal I, Winblad B, eds. Proceedings of the 9th International Conference on Alzheimer's Disease and Related Disorders Philadelphia, Pennsylvania: Alzheimer's Association; 2004: 46-58.

11. Fitzpatrick AL, et al. Midlife and late-life obesity and the risk of dementia: Cardiovascular health study. Arch Neurol. 2009;66(3):336-342.

12. Petersen RC, et al. Alzheimer's Disease Neuroimaging Initiative (ADNI): clinical characterization. Neurology. 2010;74(3):201-209.

13. McKhann G, et al. Clinical diagnosis of Alzheimer's disease: report of the NINCDS-ADRDA Work Group under the auspices of department of health and human services task force on alzheimer's disease. Neurology. 1984;34(7):939-944.

14. Landau SM, et al.; Alzheimer's Disease Neuroimaging Initiative. Amyloid- $\beta$ imaging with Pittsburgh compound B and florbetapir: comparing radiotracers and quantification methods. J Nucl Med. 2013;54(1):70-77.
15. Landau SM, et al.; Alzheimer's Disease Neuroimaging Initiative. Comparing positron emission tomography imaging and cerebrospinal fluid measurements of $\beta$-amyloid. Ann Neurol. 2013;74(6):826-836.

16. Vanderstichele H DMG SF, et al. Biomarkers for Early Diagnosis of Alzheimer's Disease. Hauppauge, NY: Nova Science Publishers; 2008.

17. Shaw LM, et al.; Alzheimer's Disease Neuroimaging Initiative. Cerebrospinal fluid biomarker signature in Alzheimer's disease neuroimaging initiative subjects. Ann Neurol. 2009;65(4):403-413.

18. Hernán MA, et al. Causal knowledge as a prerequisite for confounding evaluation: an application to birth defects epidemiology. Am J Epidemiol. 2002;155(2):176-184.

19. Lee PH. Should we adjust for a confounder if empirical and theoretical criteria yield contradictory results? A simulation study. Sci Rep. 2014;4:6085.

20. Jennrich RI, et al. Unbalanced repeated-measures models with structured covariance matrices. Biometrics. 1986;42(4):805-820.

21. Ten Have TR, et al. Mixed effects logistic regression models for longitudinal binary response data with informative drop-out. Biometrics. 1998;54(1):367-383.

22. Spira AP, et al. Objectively measured sleep and beta-amyloid burden in older adults: a pilot study. SAGE Open Med. 2014;2.

23. Sharma RA, et al. Obstructive sleep apnea severity affects amyloid burden in cognitively normal elderly: a longitudinal study. Am J Respir Crit Care Med. 2018;197(7):933-943.

24. Ju YE, et al. Obstructive sleep apnea decreases central nervous system-derived proteins in the cerebrospinal fluid. Ann Neurol. 2016;80(1):154-159.

25. Kalaria $\mathrm{RN}$, et al. Accumulation of the beta amyloid precursor protein at sites of ischemic injury in rat brain. Neuroreport. 1993;4(2):211-214.

26. Zhang X, et al. Hypoxia-inducible factor 1alpha (HIF-1alpha)mediated hypoxia increases BACE1 expression and betaamyloid generation. J Biol Chem. 2007;282(15):10873-10880.

27. Hou $\mathrm{H}$, et al. Association of obstructive sleep apnea with hypertension: a systematic review and meta-analysis. $J$ Glob Health. 2018;8(1):010405.

28. Lurie A. Endothelial dysfunction in adults with obstructive sleep apnea. Adv Cardiol. 2011;46:139-170.

29. Nagayoshi $M$, et al. Obstructive sleep apnea and incident type 2 diabetes. Sleep Med. 2016;25:156-161.

30. Mosconi L, et al. Metabolic interaction between ApoE genotype and onset age in Alzheimer's disease: implications for brain reserve. J Neurol Neurosurg Psychiatry. 2005;76(1):15-23.

31. Bero AW, et al. Neuronal activity regulates the regional vulnerability to amyloid- $\beta$ deposition. Nat Neurosci. 2011;14(6):750-756.

32. Kiviniemi V, et al. Ultra-fast magnetic resonance encephalography of physiological brain activityglymphatic pulsation mechanisms? J Cereb Blood Flow Metab. 2016;36(6):1033-1045.

33. Schlosser Covell GE, et al. Disrupted daytime activity and altered sleep-wake patterns may predict transition to mild cognitive impairment or dementia: a critically appraised topic. Neurologist. 2012;18(6):426-429.

34. Varga AW, et al. Reduced slow-wave sleep is associated with high cerebrospinal fluid a $\beta 42$ levels in cognitively normal elderly. Sleep. 2016;39(11):2041-2048. 
35. Xie L, et al. Sleep drives metabolite clearance from the adult brain. Science. 2013;342(6156):373-377.

36. Pase MP, et al. Sleep architecture and the risk of incident dementia in the community. Neurology. 2017;89(12):1244-1250.

37. Dreha-Kulaczewski S, et al. Identification of the upward movement of human CSF in vivo and its relation to the brain venous system. J Neurosci. 2017;37(9):2395-2402.

38. Green RC, et al. Depression as a risk factor for Alzheimer disease: the MIRAGE study. Arch Neurol. 2003;60(5):753-759.

39. Donohue $\mathrm{MC}$, et al.; Australian Imaging, Biomarkers, and Lifestyle Flagship Study of Ageing; Alzheimer's Disease Neuroimaging Initiative; Alzheimer's Disease Cooperative Study. The preclinical Alzheimer cognitive composite: measuring amyloid-related decline. JAMA Neurol. 2014;71(8):961-970.

40. Edmonds EC, et al.; Alzheimer's Disease Neuroimaging Initiative. Patterns of cortical and subcortical amyloid burden across stages of preclinical Alzheimer's disease. J Int Neuropsychol Soc. 2016;22(10):978-990.

41. Snowdon DA, et al. Brain infarction and the clinical expression of Alzheimer disease. The Nun Study. JAMA. 1997;277(10):813-817.

42. Cirrito JR, et al. Synaptic activity regulates interstitial fluid amyloid-beta levels in vivo. Neuron. 2005;48(6):913-922.

43. Nir Y, et al. Regional slow waves and spindles in human sleep. Neuron. 2011;70(1):153-169.

44. Ju YS, et al. Obstructive sleep apnea treatment, slow wave activity, and amyloid-beta. Ann Neurol. 2019;85(2):291-295.

45. Hosselet J, et al. Classification of sleep-disordered breathing. Am J Respir Crit Care Med. 2001;163(2):398-405.

46. Hosselet JJ, et al. Detection of flow limitation with a nasal cannula/pressure transducer system. Am J Respir Crit Care Med. 1998;157(5 Pt 1):1461-1467.

47. JuYS, etal.Slowwavesleep disruptionincreasescerebrospinal fluid amyloid- $\beta$ levels. Brain. 2017;140(8):2104-2111.

48. Osorio RS, et al. Interaction between sleep-disordered breathing and apolipoprotein $\mathrm{E}$ genotype on cerebrospinal fluid biomarkers for Alzheimer's disease in cognitively normal elderly individuals. Neurobiol Aging. 2014;35(6):1318-1324.

49. Heneka MT, et al. Neuroinflammation in Alzheimer's disease. Lancet Neurol. 2015;14(4):388-405.

50. Rosenzweig I, et al. Sleep apnoea and the brain: a complex relationship. Lancet Respir Med. 2015;3(5):404-414.

51. Jack CR, Jr., et al.; Alzheimer's Disease Neuroimaging Initiative. Serial PIB and MRI in normal, mild cognitive impairment and Alzheimer's disease: implications for sequence of pathological events in Alzheimer's disease. Brain. 2009;132(Pt 5):1355-1365.

52. Osorio RS, et al.; Alzheimer's Disease Neuroimaging Initiative. Sleep-disordered breathing advances cognitive decline in the elderly. Neurology. 2015;84(19):1964-1971.

53. Jack CR, Jr., et al.; Alzheimer's Disease Neuroimaging Initiative. Brain beta-amyloid measures and magnetic resonance imaging atrophy both predict time-toprogression from mild cognitive impairment to Alzheimer's disease. Brain. 2010;133(11):3336-3348.

54. Johnson KA, et al. Brain imaging in alzheimer disease. Cold Spring Harb Perspect Med. 2012;2(4):a006213.

55. Van Den Berg JF, et al. Disagreement between subjective and actigraphic measures of sleep duration in a populationbased study of elderly persons.J Sleep Res. 2008;17(3):295-302.

56. Unruh ML, et al. Subjective and objective sleep quality and aging in the Sleep Heart Health Study. J Am Geriatr Soc. 2008;56(7):1218-1227.

57. Young $\mathrm{T}$, et al. The occurrence of sleep-disordered breathing among middle-aged adults. $N$ Engl J Med. 1993;328(17):1230-1235.

58. Heinzer R, et al. Prevalence of sleep apnoea syndrome in the middle to old age general population. Lancet Respir Med. 2016;4(2):e5-e6.

59. Carvalho DZ, et al. Association of excessive daytime sleepiness with longitudinal $\beta$-amyloid accumulation in elderly persons without dementia. JAMA Neurol. 2018;75(6):672-680. 